\title{
Energy Constraints of Localization Techniques in Wireless Sensor Networks (WSN): A Survey
}

\author{
Griffith S. Klogo \\ Kwame Nkrumah University of Science and \\ Technology, KNUST \\ Department of Computer Engineering \\ Kumasi, Ghana
}

\author{
James D. Gadze \\ Kwame Nkrumah University of Science and \\ Technology, KNUST \\ Department of Electrical/Electronic Engineering \\ Kumasi, Ghana
}

\begin{abstract}
Wireless Sensor Network (WSN) localization is an important and fundamental problem that has received a lot of attention from the WSN research community. Determining the absolute and relative coordinate of sensor nodes in the network adds much more meaning to sense data. The research community is very rich in proposals to address this challenge in WSN. This paper, explores the various techniques proposed to address the acquisition of location information in WSN. The paper also evaluate the performance of these techniques based on the energy consumption, the skill and man hours needed to implement the technique and localization accuracy (error rate) and discuss some open issues for future research.
\end{abstract}

\section{Keywords}

Wireless sensor network, sensor nodes, localization centralized and distributed algorithm, range-based and rangefree algorithm.

\section{INTRODUCTION}

Wireless sensor networks consist of dispersed autonomous sensors that monitor physical conditions such as air pollution, water quality, heat, pressure, temperature, etc. in an environment (outdoor or in-door). Recent advancements (Micro-Electro-Mechanical Systems (MEMS) and VLSI technology) in manufacturing of small and low cost sensor nodes has led to the large scale deployment of disposable sensor nodes, especially in many applications that require unattended operations and in harsh environments. The data collected from these autonomous sensor nodes are processed into meaningful information, in the case where each sensor node is equipped with processing capabilities (Distributed Processing) or the data is transmitted to a Base Station (BS) for processing, in which case the BS has all the processing capabilities on the network (Centralized Processing).

In most Wireless Sensor Network applications (WSN), based on the mode of deployment the exact locations of the sensor nodes may be unknown. Wireless sensor network localization is an active research area and has attracted significant research interest. This will grow further with the spread of diverse wireless sensor network applications. Finding the positional coordinates of these sensor nodes manually or automatically is the process of localization [1] [3]. Location information plays an important role in making sensed data geographically meaningful and a lot more network functions can be improved based on our understanding of sensed data and location information. Sensed data and location information will help in improving network routing, topology control, congestion control, coverage, boundary detection, clustering, etc. Sensor location information can be used for tagging sensory data, which is important for application such as smart grid, environmental monitoring, etc. Determining the location (manually or automatically) of sensor nodes requires considering factors (cost, power, memory, communication cost, etc.) that directly affect the overall performance of the network. Future data dependent applications of wireless sensor network (WSN) will heavily depend on the location information provided by the network.

WSN localization is an active research area that has seen a lot proposal in terms of algorithms and techniques. Localization can be done in two ways: Centralized and Distributed [2]. Centralized localization techniques estimates the location of each sensor node on a network from a central Base Station (BS) or Server, while in the distributed localization techniques each sensor node is equipped with capabilities to estimate its own location on the network. Localization may involves determining the distance (ranging) between nodes and/or finding absolute or relative coordinates (positioning) with or without a reference node usually called the anchor (beacon) node [2].

\subsection{Performance Factors}

Irrespective of the application area of the WSN localization technique, there are some requirements that the technique should satisfy:

\subsubsection{Operation Conditions}

The technique should be clear on where it is applicable, as to whether it can work for outdoor, indoor, underwater and underground conditions. Techniques that will make use of anchor nodes equipped with GPS may not work indoors, underground and underwater since they will require line of sight with GPS satellites to determine their location. Weather and harsh environmental conditions may also affect the accuracy of the localization technique. Most algorithms cannot work well in anisotropic networks (dependent on direction), where there are various obstructions among the sensor nodes in the network. [4] Proposed a range free algorithm to minimize the localization error even in the presence of obstacles in the network.

\subsubsection{Power/Energy Efficiency}

Power is not a luxury in WSN and for that matter all localization techniques should have high efficiency in power usage. To increase the network lifetime, energy usage must be managed in every hardware and software technique [5]. Sensor nodes use energy in order to carry out three main functions: data acquisition, processing and communication. 


\subsubsection{Data Acquisition}

Depending on the type of monitoring being carried out, the energy consumed during the acquisition of data is usually negligible. Nevertheless, it may vary in considerable proportion in some applications and the regularity of acquiring the data. Distributed localization algorithms running on mobile nodes will consume more energy compared to nodes that are immobile, since mobile nodes will have to estimate their location occasionally, while immobile nodes will estimate their location once in the lifetime.

\subsubsection{Data Processing}

In distributed processing algorithms, each sensor node in the network is equipped with processing capabilities which include data processing and location estimation. While centralized processing algorithms process data and estimate location at the Base Station or Cluster-head. Hence, energy consumption on the part of each sensor node is high for distributed algorithms as compared to centralized algorithms.

\subsubsection{Data Communication}

By far most research has stated that communication consumes much more energy compared to computation [5] [6] [7] [8]. Data communication is responsible for the greatest bulk of the energy use when compared with data sensing and processing. The distance between sensor nodes also increases the energy consumption of the nodes for internode communication; however the overall energy consumption reduces with optimally placed sensor nodes [5]. Alternatively, communication would be desirable in the short range instead of long-range communication between sensor nodes. This will mean that more sensor nodes will have to be deployed for a wide coverage of an area of interest.

\subsubsection{Accuracy and Security}

Data integrity and reliability is a major issue that has attracted a lot research in Wireless Sensor Network (WSN) [9] [10] [11]. Implementation of network functions such as routing, localization, clustering, topology control, coverage, boundary detection, etc. should not compromise the availability, integrity and reliability of data in the network.

\subsubsection{Memory}

Memory is limited on small, low cost disposable sensor nodes used in many wireless Sensor Network applications. Memory is constrained in sensor networks and its management is operating system platform dependent. Most operating systems used on sensor networks such as TinyOS are event driven which are efficient in terms of time and space. A typical sensor node may only have a simple CPU and a few kilobytes of RAM, however the software running on it can take tens of thousands lines of code to implement, executing various tasks related to data acquisition (sensing), localization, wireless routing, power management, data processing (distributed processing) and network management [12].

\section{SIGNAL ATTRIBUTES}

The nature of signals used in the localization process can impair the accuracy of the technique. Signals behave differently with respect to environmental factors such as humidity, temperature, wind speed, etc. Choosing a sufficient signal type for localization depends on various factors such as node hardware, the application and the environment. Different applications and environment have various effects on the performance of location determination techniques since these techniques depend on signals. In humid air, for example, acoustic signals perform better than radio signals since moisture absorbs and reflects high frequency radio, while it has little or no effect on acoustic signals. Luckily, the loss in signal strength is logarithmic and not linear; thus the amplitude does not decrease as much in a second segment of equal length as it decreases in the first segment. Applications also put different constrains on the choice of signal type.

\section{CLASSIFICATION OF LOCALIZATION TECHNIQUES}

Wireless Sensor Network research community has proposed various localization schemes to address the need for location information that adds more meaning to sensed data in the network. Broadly, these schemes can be classified on the basis of being centralized on a single node which can be a central Base Station (BS) or a cluster head in a clustered network. The schemes could also be distributed on each sensor node in the network. Based on the measuring technique employed to acquire the absolute or relative coordinate of the sensor nodes, we could further classify these schemes as being Range-Based or Range free. Further classification of these schemes will be on the basis of the schemes estimating locational coordinates of unknown sensor nodes (static or mobile) relative to other sensor nodes with known location, usually called Anchor (Beacon) node. Such schemes are referred to as Anchor-Based or Anchor free if the technique involves estimating the location of the unknown sensor node in reference to an anchor node or without reference to an anchor node respectively. Localization schemes could involve a combination of the aforementioned schemes.

\subsection{Centralized localization}

Centralized localization algorithm requires a central sensor node or Base Station (BS) with unlimited computational power and energy. The base station gathers network wide information for localization. Base Station (BS) determines the location of each node by collecting data and transmits them back into the network. Data is gathered globally from the network by message exchange between nodes, hence, with the increase in the number of sensor nodes in the network; centralized location algorithm may be prone to energyinefficiency, delay and bottlenecks in network traffic. This phenomenon will be much more prevalent in cases where sensor nodes are mobile and location information will have to be computed occasionally to estimate the location of mobile nodes at any point in time. Centralized localization may have inherent errors if sensor nodes depend on other nodes to route their data or information to the Base Station (BS). Centralized localization techniques will work effectively on small networks with each node interacting directly with the Base Station (BS). The accuracy in this case will be high compared to other implementation techniques, due to the availability of global information on the network and the high computation and energy available to the Base Station. One important proposal presented in the area of centralized localization is Multidimensional scaling (MDS-MAP). There are also some other techniques proposed by the Wireless Sensor Network (WSN) community based on Semi-Definite Programming (SDP), Simulated Annealing and Received Signal Strength Indicator (RSSI).

\subsection{Distributed localization}

A distributed localization algorithm decentralizes computation to most sensor nodes in the network. Each sensor node is configured to estimate its location with or without reference to some anchor nodes in the network. This algorithm usually 
employs inter node communication for estimating the location of the sensor nodes. Inter node communication consumes less energy as compared to the energy consumption in the case of centralized algorithms. It is usually hard to implement but has high flexibility and computational efficiency for large scale deployed sensor networks. Distributed localization is a research area that is still largely unexplored. In this class of algorithms there is no notion of a hub center that collects information from other sensors. Instead, sensors exchange information to localize the source in a distributed manner. A distributed implementation based on second order cone programming (SOCP) was proposed in [13], where both inaccuracies in anchor positions and distance measurements are considered to refine the position estimation of the algorithm. [14] also proposed using a single moving beacon in a distributed manner for sensor nodes to compute their position estimates based on the range-free technique. Energy efficiency is a major concern in distributed algorithm implementation, in an effort to address this, [15] proposed an energy-efficient distributed parameter estimation method for applications in wireless sensor networks. The estimation approach is based on an incremental realization of GaussNewton method.

\section{RANGE FREE AND RANGE-BASED}

A basic building block of most localization techniques is ranging. Ranging is a process or method to determine the distance from one location or position to another location or position. In wireless sensor networks localization, rangebased techniques rely on nodes measuring distances or angles to reference nodes usually called anchor nodes while rangefree techniques do not require nodes measuring distances or angles to reference nodes.

\subsection{Range-Based Localization Techniques}

Range-Based techniques require direct measurement of distances and may not have specific topological requirements apart from simple geometric collinearity constraints (i.e., the angle between each node must be greater than $\left.\left(0^{0}\right)\right)$. This technique depends on measured distance or angle between nodes to estimate the location of unknown or mobile nodes in the network. The technique starts with estimating the distance or angle to each sensor node. There have been several research proposals on range-based techniques, some of which include AOA, TOA, TDOA and RSSI. After acquiring the distance or angles of unknown nodes with reference to anchor nodes, the absolute or relative position of the unknown node is determined by Tri/Multi-lateration.

\subsubsection{Angle of Arrival (AOA) / Degree of Arrival (DOA)}

AOA based positioning techniques involve measuring angles of the node seen by reference nodes. AOA measurement is a method for determining the direction (Degree of Arrival) of propagation of a radio-frequency wave for example of an antenna. Based on the positioning of the sensor nodes, the Degree of Arrival (DOA) of the emitting source node is estimated. With the measured angles, each sensor node is able to calculate the position of other nodes or determine the angle of the unknown node's relative position to some anchor node. Basic trigonometry relations are employed in determining the angles of source nodes to the normal (anchor node) in the network. Angle of Arrival (AOA) estimation is fundamental to many wireless sensing and communication applications, especially RF source localization. AOA is typically performed using antenna array elements that are mapped to the incident direction of the signals to a reference node. There are two advantages of using AOA, first the phase of the received signal at each node is usually more stable than the received signal strength (RSS) and secondly, for an effective AOA estimation scheme, two antenna arrays will achieve accurate node localization as compared to other range-based approaches that will require three or more sensor nodes. Generally, AOA technique provides more accurate localization result than RSSI based techniques but the cost of hardware is high in AOA. AOA is prone to effects such as multi-path effects (two or more paths of a signal arriving at a receiving antenna), shadowing, scattering and also when there is no line of sight. A distributed AOA-based localization and orientation approach for wireless sensor networks under the assumption that all unknown sensor nodes are capable of detecting angles of the incident signal from neighboring nodes is proposed in [16]. Other AOA localization techniques can be seen in [17] [18], employing L-shaped minimum redundancy linear array antennas equipped at anchor nodes and RFID Tags. This technique provides a high degree of accuracy for localization when there a clear line-of-sight between nodes in the network. The technique also requires skills and man hours in setting up the antennas to provide the required spacing for spatial diversity to accurately estimate the AOA.

\subsubsection{Time of Arrival (TOA)/Time of Flight (TOF)}

Time of Arrival (TOA) is the time taken for a radio signal to travel from a single transmitter to a remote single receiver. TOA employs the relation between light speed in a vacuum and the carrier frequency of a signal the time is a measure for the distance between transmitter and receiver. TOA uses the absolute time of arrival at a sensor node rather than the measured time difference between departing from one node and arriving at the other nodes in the network. The distance between two sensor nodes can be directly calculated from the time of arrival as signals travel with a known velocity. The TOA can be estimated either by measuring the phase of the received narrowband carrier signal or by direct measurement of the arrival time of a wideband narrow pulse. The propagation time can be directly translated into distance, based on the known signal propagation speed (velocity). Techniques based on time-of-arrival (TOA) measurements are the most promising since they can offer an appropriate balance between accuracy and complexity. This method can be applied to different signal types including RF, Acoustic, Infrared and Ultrasound. With a global clock, a receiving node can estimate the TOA of an incoming signal (usually time-stamped by the reference node) from other neighboring nodes in the network. TOA method requires synchronization of sensor nodes in the network. Conventional TOA-based localization algorithms are typically designed for single events. [20] proposed a computationally efficient framework for utilizing Time of Arrival (TOA) sensor to localize multiple events in close proximity in space and time. This technique apart from high accuracy in clear line-of-sight does require round-trip propagation of signals to estimate the distance between nodes, this puts extra communication cost on the nodes increasing the power usage by the RF devices. Synchronization also means extra hardware leading to power usage.

\subsubsection{Time Difference of Arrival (TDOA)}

TDOA calculates the location of sensor nodes by using difference in the time of arrival of signals at three or more receiving sensor nodes. This technique can locate a sensor node by accurately computing the time difference of arrival of the signal emitted from transmitting node to three or more 
neighboring sensor nodes in the network. It also refers to the case of locating sensor nodes that are synchronized in a network. Three key features or necessary functions needed to localize sensor nodes by TDOA method are ranging, communication and synchronization. All sensor nodes in the network need to be synchronized for accuracy in ranging measurements. This technique is dependent on the accuracy of synchronization. There have been several research proposals using TDOA, some employing Ultra Wideband (UWB) enhanced TDOA technique [19]. UWB transceivers (radios) are used because of its ability to wireless synchronize multiple RF receivers. The UWB link can carry data for two-way communication, and provide fast accurate ranging in line-ofsight (LOS). Implementing this technique in both multi-node and multi-signal mode require extra hardware which adds more strain on the power available.

\subsubsection{Received (Radio) Signal Strength (RSS)}

RSS based localization is by far a very popular technique employed by the wireless sensor network community. This technique involves measuring the power of an incoming signal at a receiving node, based on the known transmitted power; the effective propagation loss can be calculated. Theoretical and empirical models are used to translate the loss in measured power into distance estimate for localization. In many ways, radio signal strength (RSS) is popular and an ideal modality for range estimation of sensor nodes in wireless networks because its use come at no additional cost in setting up the network. RSS is especially appealing for localization due to its simplicity in implementation that does not introduce additional cost, energy and size constraints into the network. Despite its appealing nature, RSS does yield very noisy range estimations since it suffers from reflection and attenuation in the environment of its application [21]. These effects can have much larger impact than loss of signal strength as a result of travelling longer distance, making it difficult to infer from measure losses without a very good model of the environment. Due to the relatively high noise in measurement, there are proposals to reduce the margin of error that will reduce the extent to which the measurement errors that affect the estimation of the absolute or relative positional coordinate of the sensor nodes in the network. There have been several proposed techniques using RSS information without the need for timing and synchronization, some of them being formulated in the context of maximum likelihood (ML) [23]. Formulation for RSS based localization in the context of maximum likelihood is evidently nonlinear and non-convex. Linear least square (LLS), multidimensional scaling (MDS) and semidefinite programming (SDP) relaxation are methods that have been proposed to solve the problems of localization, where the SDP method has been found to give the best performance with higher computational complexity [22].

Using RSS based localization means the following factors must be considered;

1. Channel Characteristics: RSS based positioning techniques are very sensitive to channel parameters estimations such as relative delay, Doppler frequency etc.

2. Signal Modality: Different signal have different attributes when it comes to reflection, scattering, fading and attenuation.

3. Environmental factors: RSS is highly sensitive to subtle environmental factor such as grass height, radio enclosure and elevation of sensor nodes from the ground [21].

The advantage of using this technique comes with no extra hardware and memory, but the technique suffers from usual
RF challenges like multi-path effects and shadowing hence moderate accuracy in clear line-of-sight (LOS) and very poor in Non line-of-sight (NLoS). To improve this technique involves pre-deployment calibration to model channel parameters which will add to the total cost of implementing the technique.

\subsubsection{RSS profiling/Fingerprints}

Radio Fingerprinting is a process that identifies a sensor node uniquely by its "fingerprint" that characterizes its signal transmission in the network. Radio Fingerprinting also known as Signature Database or Pattern Matching or Database Correction is also an approach to localizing unknown sensor nodes in a network. With this technique, sensor nodes listen to radio signals from surrounding networks. The unique signal strengths are used to identify spots in the network and building a grid with the spots linking it to a map for positioning of unknown nodes. In other words, the idea is to first determine the RSS values in several points and save these values in a database and later use these values for comparison with received RSS values from the nodes with known locations in the network for position estimation. This technique suffers from multipath interference which is typical with wireless signals as a result of bouncing off objects on route to its destination. To employ this system, a beacon node must send test signals around to various locations so the nodes can record the fingerprints and create a database for comparison later on. To get more accurate and comprehensive results, measurement with this technique is taken with reference to nodes with known locations in the network. The accuracy of this technique is bounded due to the random environmental changes which cause inconsistency between the stored fingerprints and the current radio behavior. [24] proposed a cooperative localization scheme, whereby users exchange their real-time signal measurement in order to update and improve their estimated location. This technique requires sensor nodes keeping a database of fingerprints which can put constraints on the limited memory available for these nodes. Despite the constraints on memory, this technique is software based and will require no new hardware, spectrum, or sensing technologies outside of what already exists in the network. This technique also has lower power consumption compared to GPS.

\subsection{Range Free Technique}

Range-free approaches for localization are based on sensor nodes wireless connectivity and topology to estimate the location of unknown sensor nodes [25] [26]. These techniques provide advantages of low cost, small communication traffic, no extra hardware and provide some degree of precision to localization, sometimes even better than some range based methods. However, a large number of static anchor nodes (nodes with known coordinates) and specific deployment is required for a high degree of accuracy, thereby increasing the cost of implementing this method [27].

\subsubsection{Binary Proximity}

This is a very basic location technique, binary proximity involves a simple decision of whether some sensor nodes are within the reception range of each other or not. This technique usually employs some anchor nodes as reference for locating unknown nodes in the network. These anchor nodes usually transmit beacon signals which the unknown nodes use to estimate their position in the network. Alternatively, unknown nodes will transmit beacon signals as and when it needs to be localized. This technique is most like to be efficient for 
clustering in a large scale network. An excellent example of proximity detection as a means for localization is the Active Badge location system meant for an indoor office environment [28]. A much larger application of localization, using binary proximity detection, is with passive radio frequency identification (RFID) tags. RFIDs are employed for animal tracking and environmental monitoring. A key difference in RFID proximity detection compared with active badge is that the unknown nodes are passive tags being queried by reference nodes in the sensor network [29]. No extra hardware is required in this technique but relies heavily on anchor nodes for accuracy in clear line-of-sight and RF (signal) coverage which may pose challenges for some applications.

\subsubsection{Centroid}

Proximity information gathered globally or locally depending on the size of the network can be used to greater advantage when the density of reference nodes in sufficiently high that there are several reference nodes within the unknown node [29] [30]. An unknown node within the reception range of a group of reference nodes can estimate its location with ease. The algorithm implementation contains two core steps. First, all anchors broadcast their position to all nodes in the network within the transmission or reception range. Each unknown node listens for a fixed period of time and collects all beacon signals it receives from various reference points. Secondly, all unknown nodes estimate their location by centroid determination with the proximity information gathered from the anchors nodes. This technique is simple but dependent on anchor nodes, which can introduce some error in location estimation with minimum number of the anchor nodes. To solve the problem of localization error [31] proposed an enhanced centroid localization method using edge weight of adjacent reference nodes based on TSK fuzzy modeling. Besides, the conventional centroid localization algorithm only focuses on node self-localization for 2D networks. [30] proposed a 3D positioning algorithm for WSN based on linear relationship between rectangular and volume coordinates. This technique also depends heavily on anchor nodes and the proximity of the signal used. It also involves unknown nodes listening to anchor signals for some random time which adds to power usage.

\subsubsection{Geometric Constraints}

Radio and other signal coverage for a given sensor node in a network can be described by some geometric attributes. The limits on these signal characteristics or shapes can be used to provide relative location estimates of sensor nodes by determining the geometric regions the node is constrained in, because of intersections between overlapping coverage regions. For instance, the area of radio coverage may have upper-limits of a circle of radius say $R_{\max }$. In other word, if a node $\mathrm{B}$ hears node $\mathrm{A}$, it knows that it must be no more than distance say $R_{\max }$ from node $A$. So if an unknown node hears from several reference nodes, it can determine that it must lies in the symmetrical area described by the connection of circles of radius $R_{\max }$ which is centered on these nodes [29]. High accuracy is assured due to the high centralized nature of this technique. Location information needs to be disseminated to each sensor node in the network. This requirement can contribute significantly to increasing the communication overheads and energy consumption of the algorithm. Failure of a single node will necessitate frequent information exchange throughout the network adding to the communication and energy cost.

\subsubsection{Approximate Point in Triangulation (APIT)}

An approach similar to localization using geometric constraint is the approximate point-in-triangulation (APIT) technique. APIT is similar to geometric constraint localization method in that it provides location estimate as the centroid of an intersection of regions. The novelty in the implementation of this technique lies in how the regions are defined. Triangles between different sets of three reference nodes (rather than the coverage of a single node) are used in the localization procedure. APIT employs local area approach, where anchor nodes demarcate the network of nodes into triangular regions. A node's presence inside or outside of these triangular regions allows a node to narrow its area in which it can potentially reside. It is usually very difficult to locate unknown nodes lying within the triangle formed by three reference nodes if the nodes are stationary since wireless signal propagation is usually not ideal. An approximate solution can be determined using some prior location information, that is, the ability to determine which of two nodes is nearer a third node based on the signal reception. One limitation for the APIT technique is that it can provide inherent erroneous results, because the determination of whether a node lies within a particular triangle requires quite a high deployment of anchor nodes in order to provide good localization accuracy [29]. This technique depends on anchor nodes but has the advantage of no extra hardware. Localization error of $0.4 \mathrm{R}$ is acceptable, where $\mathrm{R}$ is the range of the anchor nodes [33].

\subsubsection{Distance Vector Hop (DV-Hop)}

DV-Hop localization is a distributed, hop-to-hop positioning algorithm. Anchor nodes generate packets including their position information and a flag which is initialized as one to figure the number of hops away from them. These packets are broadcasted into WSN. When they are transmitted by the intermediate nodes, the hop number is increased by one. In this way, any node can determine the hop number from it to a certain anchor node. Similarly the anchor nodes can compute their hops to other anchors as well. The average distance for each hop can be computed by a simple formula and then will be broadcasted. When an unknown node receives it, the receiver will estimate its distance to the anchor node. The location can be figured out by trilateration [32] [33] [34] [35], after an unknown node obtains three or more estimated values from anchor nodes. Position of anchor nodes also has a major effect on the localization accuracy [32] in the implementation of this technique. Most proposals using this technique assume a network where all nodes are in clear line of sight with each other, but in real applications nodes may have obstacles between them making the signal go round these obstacles which might not give clear indication of the actual hop count distance between an anchor node and an unknown node. To solve the problem [32] proposed a selective anchor node localization algorithm (SANLA). It is also evident that the number of anchor nodes does not necessarily increase the precision of the localization technique when using hop count. This technique involves internode communication and depends on anchor nodes. It has high accuracy in clear lineof-sight to the anchor nodes, but localization can be erroneous when there is no line-of-sight to anchors. It does not require any extra hardware.

\subsubsection{ROCRSSI}

Ring Overlapping based on Comparison of Received Signal Strength Indicator (ROCRSSI) is another range-free localization approach proposed recently, this technique aims at dealing with the undetermined node problem of APIT and 
achieving higher estimation accuracy with lower communication overhead. However, in [36], only a rough comparison on the performance of APIT and ROCRSSI is provided. In [37] a more detailed simulation results is presented to demonstrate that ROCRSSI effectively alleviates the "undetermined node problem and outperforms APIT in terms of achievable estimation accuracy and energy efficiency. Furthermore, system configurations that directly affect the performance of ROCRSSI are investigated thoroughly, and the achievable estimation accuracy under different system configurations is presented as guidelines of deciding system parameters when using ROCRSSI for sensor localization. If all directional antennas are used in a consistent environment, an isotropic radio attenuation model can be assumed and ROCRSSI can get the best estimation accuracy. Although ROCRSSI depends on the consistency of radio transmission in a wide range of directions, the estimated location errors by ROCRSSI are generally small even under circumstances with very irregular radio propagation [36]. This technique has a better accuracy compared to APIT and has low localization error in both line-of-sight and Non line-ofsight. This technique depends on RF signal strength and the configuration of its antennas which adds to the power consumption of the nodes in the network.

\subsection{Comparison of localization Techniques}

Table 1 below, is a comparative assessment on all the localization techniques discussed above based on the hardware requirements, dependence on anchor nodes, line-ofsight (LOS) and Non line-of-sight (NLoS) accuracy, localization error, power consumption and communication cost.

\section{ANCHOR FREE AND ANCHOR BASED}

Anchor-based localization involves determining the location of an unknown node (fixed or mobile) with respect to a node with known location called Anchor node [32] [38] [39] [40], while Anchor free is without reference to an anchor node. Research has established that the presence of anchors in the network for localization does increases the degree of accuracy in estimating the positional coordinates of the unknown node(s) Anchor nodes are those nodes in the network that already know their location by means of direct placement at know coordinates, hard coding the location or acquired by using localizing systems such as a Global Positioning System (GPS) receiver. Deploying anchor nodes is both labour and hardware intensive hence the need for anchor-free localization techniques. Since sensor nodes are deployed randomly via air planes into unfriendly terrains or harsh environments for environmental monitoring and military surveillance, there is the need for algorithms that may not necessarily depend on anchor nodes for localization.
There is the need for optimal localization algorithms that will not depend on anchors for the following reasons:

a. The use of small, low cost and disposable sensor nodes.

VLSI technology and advancement in the manufacturing of Micro-Electro-Mechanical Systems (MEMS) has enabled wide application areas of wireless sensor networks. The small, low cost and disposable nature of these sensor nodes has also informed large scale deployment and wide coverage of these nodes in harsh and unfriendly terrains for various environmental monitoring and military applications.

b. Labour and risk in placing anchor nodes

Anchor nodes with hard coded location coordinates will have to be placed at the right position in the network. This may not be possible in some harsh environmental monitoring applications and will be at a high cost if it can be done.

c. Global Positioning System (GPS) coverage

Underground, underwater and some plane area above sea level are unreachable for GPS usage. Hence applications that will depend on GPS to estimate the location of anchors may be prone to errors since the localization results are directly determined by GPS accuracy.

\subsection{Anchor Placement}

Most researchers have said that anchors are best when placed uniformly on the perimeter of the network [41] [42]. Nonetheless, it may not be the case for all techniques, since some researchers have also proposed otherwise [32] [41]. It is important to find optimal position for anchor nodes to reduce localization error and to avoid excessive deployment costs. Deployment cost of anchor nodes involves both hardware and labour. There are proposals for mobile anchor-based localization and various conditions in the network with respect to path planning and noise models [38] [39] [43].

\section{OPEN ISSUES}

Despite the extensive literature on sensor network localization, there are issues that are yet to be explored extensively and there are others that the research community is yet to take definite stand on. Some of these issues are discussed below.

\subsection{Energy/Power Constraints}

Energy/Power consumption in WSN localization has had lots of proposals addressing its usage and management, but there are more to be desired for highly optimized localizations techniques with high power efficiency. Employing energy efficient design at the communication layer and all aspects of a localization algorithm for energy constrained wireless sensor network requires developing appropriate models for accessing the energy consumption. This obviously is not a trivial task since it may cover many areas of the communication and computation of the absolute or relative location of unknown nodes in the network. 
Table 1: Comparative Study of localization techniques

\begin{tabular}{|c|c|c|c|c|c|c|c|}
\hline \multirow[b]{2}{*}{$\begin{array}{c}\text { Localization } \\
\text { Algorithms/Techniques }\end{array}$} & \multicolumn{7}{|c|}{ Performance factors } \\
\hline & 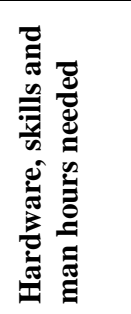 & 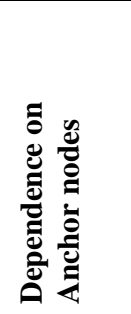 & 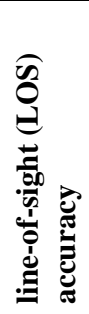 & 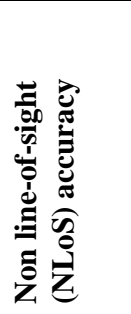 & 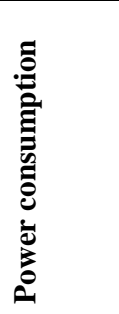 & 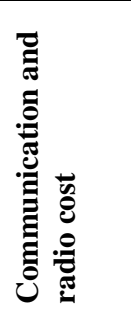 & 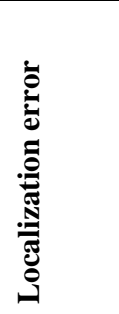 \\
\hline Angle of Arrival (AOA) & High & Medium & High & Low & Medium & Very low & Medium \\
\hline Time of Arrival (TOA) & High & Medium & High & Low & Medium & Medium & Medium \\
\hline $\begin{array}{l}\text { Time Difference of Arrival } \\
\text { (TDOA) }\end{array}$ & High & Medium & High & Low & Medium & Medium & Medium \\
\hline $\begin{array}{ll}\text { Received (Radio) } & \text { Signal } \\
\text { Strength (RSS) } & \end{array}$ & Very low & Medium & High & Very low & Low & Low & Medium \\
\hline RSS profiling/Fingerprints & High & Medium & High & Medium & Medium & Medium & Low \\
\hline Binary Proximity & low & High & High & Very low & Low & Low & Medium \\
\hline Centroid & Low & High & High & Very low & Low & Low & Medium \\
\hline Geometric Constraints & Low & High & High & Very low & Low & Low & Medium \\
\hline $\begin{array}{l}\text { Approximate Point } \\
\text { Triangulation (APIT) }\end{array}$ & Very low & High & High & Low & Low & High & Medium \\
\hline $\begin{array}{l}\text { Distance Vector Hop (DV- } \\
\text { Hop) }\end{array}$ & Low & High & High & Low & Low & High & Low \\
\hline ROCRSSI & High & High & High & Very low & High & High & Low \\
\hline
\end{tabular}

\subsection{D WSN}

Most localization techniques in WSN determine the location of unknown nodes (static or mobile) in a 2D plane/space. However, sensor nodes are mostly deployed in 3D space. There is the need then to explore location determination techniques in 3D space. Irregularities in signal attribute have been established in comparing radio frequency signals in 2D and $3 \mathrm{D}$, hence localization in techniques $3 \mathrm{D}$ will not be farfetched.

\subsection{Security and Privacy}

A major issue in large scale deployment of WSN is security. In as much as location information is important to adding meaning to sense data, it is also important that the techniques employed in determining the location does not compromise the security and privacy of the network. Security is very critical in most WSN applications such as smart OR's and military surveillance. Although some research on security of localization schemes have been proposed, they are only related to typical cases and applications. Privacy of node locations is barely mentioned in most localization processes.

\subsection{Anchors Placement Strategies}

Anchors play important role in determining absolute or relative coordinates of sensor nodes in WSN localization technique. There are some researchers proposing perimeter placement of anchor nodes while others proposed mobile, within network and random anchor node placement. These proposals brings to question the following: whether

a. Anchor node placement is technique dependent

b. Anchor node placement is application dependent

c. Anchor node placement is network size dependent

\section{CONCLUSION}

This paper dealt with localization techniques and sort to bring to light major strides that has been made in WSN localization. An assessment of these techniques was also done relative to some performance parameters especially the energy constraints that beset these techniques. Radio requirements of these techniques are major contributors to the power consumption of the sensor nodes in the network; hence the localization techniques that depend on radio heavily have high power consumption. The paper also sorts to expose some issues that are yet to receive much attention from the WSN research community. 


\section{REFERENCES}

[1] A. Savvides, M. Srivastava, L. Grod and D. Estrin, "Localiztion in Sensor Networks", University of Califonia, Los Angeles.

[2] U. Nazir, M. A. Arshad, N. Shahid and S.H. Raza, "Classification of Localization Algorithms for Wireless Sensor Network: A Survey", 2012 International Conference on Open Source Systems and Technologies (ICOSST), pp 1-5

[3] G. Mao, B. Fidan and B. D. O. Anderson, "Wireless sensor network localization techniques", Computer Networks 51 (2007) pp 2529-2553

[4] H. Chen, Q. Shi, R. Tan, H. V. Poor, Fellow, IEEE, and K. Sezaki, "Mobile Element Assisted Cooperative Localization for Wireless Sensor Network with Obstacles", IEEE transactions on wireless communications, Vol. 9, No. 3, March 2010, pp 956-963

[5] F. Shebli, I. Dayoub and J. M. Rouvaen, "Minimizing energy consumption within wireless sensor networks", Ubiquitous Computing and Communication Journal

[6] Y. Liang and W. Peng , "Minimizing Energy Consumption in Wireless Networks via Two-Modal Transmission", ACM SIGCOMM Computer Communication Review, Vol 40, Number 1, January 2010, pp 13-18

[7] A. Garcia-Saavedra, P. Serrano, A. Banchs, and G. Biances, "Energy Consumption Anatomy of 802.11 Devices and its Implication on Modeling and Design", Co-NEXT'12, December 10-13, 2012, Nice, France, pp $169-180$

[8] G. Miao, N. Himayat, Y. Li and A. Swami, "Cross-Layer Optimization for Energy-Efficient Wireless Communications: A Survey", School of Engineering and Computer Engineering, Georgia Institute of Technology, Atlanta, Georgia, 30332-0250, pp 1-13

[9] I. Kamel and H Juma, "A lightweight Data Integrity Scheme for SensorNetworks",http://www.ncbi.nlm.nih.gov/pmc/artic les/PMC3231299/, Published online 2011 April 7, pp 4118-4136

[10] I. Talzi, S Schonborn, and C. Tschudin, "Providing Data Integrity in Intermittently Connected Wireless Network", Computer Science Dept., University of Basel, Switzerland, pp1-8

[11] A. Giani, T. Roosta and S. Sartry, "Integrity checker for Wireless Sensor networks in Health Care Applications". Department of Electrical Engineering and Computer Sciences, University of California, Berkeley, pp 1-4

[12] R. Chu, L. Gu, Y. Liu, M. Li, X. Lu, "Versatile Stack Management for multitasking Sensor Network", IEEE Computer Society, 2010 International Conference on Distributed Computer Systems, pp 388-397

[13] S. Srirangarajan, Member, IEEE, A. H. Tewfik, Fellow, IEEE, and Z. Luo, Fellow, IEEE, "Distributed Sensor Network Localization Using SOCP Relaxation", IEEE Transaction on wireless Communication, Vol. 7, NO. 12, December 2008, pp 4886-4895
[14] B. Xiao, Member, IEEE, H. Chen, Member, IEEE, and S. Zhou, Member, IEEE, "Distributed Localization Using a Moving Beacon in Wireless Sensor Networks", IEEE Transactions on parallel and Distributed systems, Vol. 19, NO. 5, May 2008, pp 587-600

[15] T. Zhao, Member, IEEE, and A. Nehorai, Fellow, IEEE, "Information-Driven Distributed Maximum likelihood Estimation Based on Gauss-Newton Method in Wireless Sensor Network", IEEE Transaction on Signal Processing, Vol. 55, No. 9, September 2007, pp 46694682

[16] R. Peng and M. Sichitiu, "Angle of Arrival Localization for Wireless Sensor Networks", Department of Electrical and Computer Engineering, North Carolina State University, pp 1-9

[17] B. Yao, W. Wang and Q. Yin, "Angle of Departure Aided Sensor Localization Technique under Multipath Environment", Globecom 2012, Ad-Hoc and Sensor Network Symposium, pp 304-308

[18] J. Zhou, H. Zhang and L. Mo, "Two-dimensional Localization of Passive RFID Tags Using AOA Estimation", 978-1-4244-7935-1/11 @2011, IEEE, pp 15

[19] B. Lonske, E. V. Doorn, S. Ponnaluri and A. Bhat, "UWB Enhanced Time Difference of Arrival System", Intelligent Automation Incorporated, pp 1-32

[20] S. Venkateswaran and U. Madhow, "Space-Time Localization using Time of Arrival", Department of Electrical and Computer Engineering, University of California Santa Barbara, CA 93106, USA, pp 1-8

[21] K. Whitehouse, C. Karlof and D. Culler, "A practical Evaluation of Radio Signal Strength for Ranging-based Localization", pp 1-12

[22] G. Wang and K. Yang, Member, IEEE, "A New Approach to Sensor Node Localization Using RSS Measurements in Wireless Sensor Networks", IEEE Transaction on wireless Communication, Vol. 10, NO. 5, MAY 2011, pp 1389-1395

[23] J. Prieto, A. Bahillo, S. Mazuelas, P. Fernandez, R. M. Lorenzo and E. J. Abril, "Self-Calibration of TOA/Distance Relationship for Wireless Localization in Hasrh Environments". IEEE ICC 2012 - Ad-hoc and Sensor Network Symposium, pp 571-575

[24] K. Papapostolous, W. Xiao, and H. Chaouchi, "Cooperative fingerprint-based indoor localization using Self-Organizing Maps", Wireless Communication and Mobile Computing Conference (IWCMC), $20117^{\text {th }}$ International, pp 1814-1819

[25] Z. Zhong and T. He, Member, IEEE, "RSD: A Metric for Achieving Range-Free Localization beyond Connectivity", IEEE Transaction on Parallel and Distributed Systems, Vol. 22, NO. 11, Novermber 2011, pp 1943-1951

[26] Y. Wang, X. Wang, D. Wang, Members, IEEE and D. P. Agrawal, Fellow, IEEE, "Range-Free Localization Using Expected Hop Progress in Wireless Sensor Networks", IEEE Transaction on Parallel and Distributed Systems, Vol. 20, NO. 10, October 2009, pp 1540-1552 
[27] B. Li, Y. He, F. Guo and L. Zuo, "A Novel Localization Algorithm Based on Isomap and Partial Least Squares for Wireless Sensor Networks", IEEE Transaction on Instrumentation and Measurement, Vol. 62, NO. 2, February 2013, pp 304-314

[28] R. Want, A. Hopper, V. Falcao and J. Gibbons, "The Active Badge Location System", Olivetti Research Ltd. (ORL), Cambridge, England, pp 1-10

[29] B. Krishnamachari, "Networking Wireless Sensors", Cambridge University Press, ISBN-13: 978-0-52183847-4

[30] H. Chen, P. Huang, M. Martins, H. Cheung So and K. Sezaki, "Novel Centroid Localization Algorithm for Three-Dimensional Wireless Sensor Networks", pp 1-4

[31] S. Yun, J. Lee, W. Chung and E. Kim, "Centroid Localization Method in Wireless Sensor Network using TSK Fuzzy Modeling", Department of Electrical and Electronic Engineering, Yonsei University, Seoul, Korea, pp $1-5$

[32] S. Tian, X. Zhang, X. Wang, P. Sun and H. Zhang, "A Selective Anchor Node Localization Algorithm for Wireless Sensor Networks", 2007 International Conference on Convergence Information Technology , pp 358-362

[33] I. F. Akyildiz and M. C. Vuran, "Wireless Sensor Networks, Wiley, pp 283-284

[34] H. Wu and R. Gao, "An Improved Method of DV-hop Localization Algorithm", Journal of Computational Information Systems 7: 7 (2011), pp 2293-2298

[35] H. Chen, K. Sezaki, P. Deng and H. Cheung So, "An Improved DV-Hop Localization Algorithm for Wireless Sensor Networks", 978-1-4244-1718-6/08 @2008 IEEE, pp 1557-1561
[36] C. Liu, K. Wu and T. He, "Sensor Localization with Ring Overlapping Based on Comparison of Received Signal Strength Indicator", 0-7803-8815-1/04 (O2004 IEEE, pp 516-518

[37] C. Liu and K. Wu, "Performance Evaluation of RangeFree Localization Methods for Wireless Sensor Networks", Computer Science Department, University of Victoria, BC, Canada V8W 3P6, pp 1-8

[38] N. Salman, H. K. Maheshwari, A.H. Kemp and M. Ghogho, "Effects of anchor placement on mean-CRB for localization", 2011 The 10th IFPI Annual Mediterranean Ad Hoc Networking Workshop, pp 115-118

[39] Chia-Ho Ou, Member, IEEE and Wei-Lun He, "Path Planning Algorithm for Mobile Anchor-Based Localization in Wireless Sensor Networks", IEEE Sensor Journal, Vol. 13, NO. 2, February 2013, pp 466-475

[40] B. Xiao, Member, IEEE, L. Chen, Q. Xiao and M. Li, Member, IEEE, "Reliable Anchor-Based Sensor Localization in Irregular Areas", IEEE Transactions on Mobile Computing, Vol. 9, NO. 1, January 2009, pp 6072

[41] R. Akl, K. Pasupathy and M Haidar, "Anchor Nodes Placement for Effective Passive Localization", 2011 International Conference on Selected Topics in Mobile and Wireless Networking (ICOST), pp 127-132

[42] J. N. Ash and R. L. Moses, "On Optimal Anchor Node Placement in Sensor Localization by Optimization of Subspace Principal Angles", ICASSP C2008 IEEE, pp 2289-2292

[43] Y. Zhou and L. Lamont, "A Mobile Beacon Based Localization Approach for Wireless Sensor Network Applications", SENSORCOMM 2011: The Fifth International Conference on Sensor Technology and Applications, pp 243-248 